\title{
Identically self-blocking clutters
}

\author{
Ahmad Abdi \\ Gérard Cornuéjols \\ Dabeen Lee
}

February 4, 2019

\begin{abstract}
A clutter is identically self-blocking if it is equal to its blocker. We prove that every identically selfblocking clutter different from $\{\{a\}\}$ is nonideal. Our proofs borrow tools from Gauge Duality and Quadratic Programming. Along the way we provide a new lower bound for the packing number of an arbitrary clutter.
\end{abstract}

\section{The main result}

All sets considered in this paper are finite. Let $V$ be a set of elements, and let $\mathcal{C}$ be a family of subsets of $V$ called members. If no member contains another, then $\mathcal{C}$ is said to be a clutter over ground set $V$ [12]. All clutters considered in this paper are different from \{\}$,\{\emptyset\}$. Let $\mathcal{C}$ be a clutter over ground set $V$. A cover is a subset of $V$ that intersects every member. The covering number, denoted $\tau(\mathcal{C})$, is the minimum cardinality of a cover. A packing is a collection of pairwise disjoint members. The packing number, denoted $\nu(\mathcal{C})$, is the maximum cardinality of a packing. Observe that $\tau(\mathcal{C}) \geq \nu(\mathcal{C})$. A cover is minimal if it does not contain another cover. The family of minimal covers forms another clutter over ground set $V$; this clutter is called the blocker of $\mathcal{C}$ and is denoted $b(\mathcal{C})$ [12]. It is well-known that $b(b(\mathcal{C}))=\mathcal{C}[17,12]$. We say that $\mathcal{C}$ is an identically self-blocking clutter if $\mathcal{C}=b(\mathcal{C})$. (This terminology was coined in [4].) Observe that $\{a\}$ is the only identically self-blocking clutter with a member of cardinality one.

Theorem 1 ([6]). A clutter $\mathcal{C}$ is identically self-blocking if, and only if, $\nu(\mathcal{C})=\nu(b(\mathcal{C}))=1$.

Consider for $w \in \mathbb{Z}_{+}^{V}$ the dual pair of linear programs

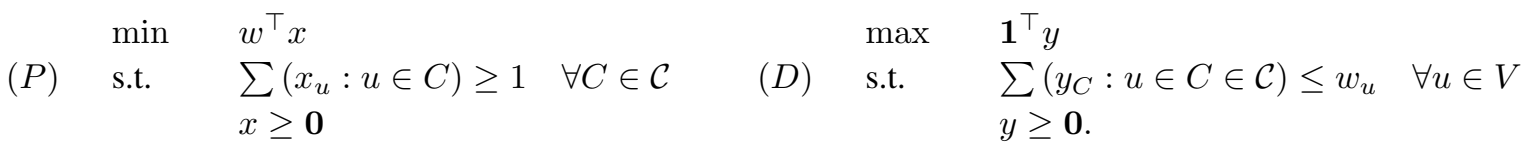

Denote by $\tau^{\star}(\mathcal{C}, w), \nu^{\star}(\mathcal{C}, w)$ the optimal values of $(P),(D)$, respectively, and by $\tau(\mathcal{C}, w), \nu(\mathcal{C}, w)$ the optimal values of $(P),(D)$ subject to the additional integrality constraints $x \in \mathbb{Z}^{V}, y \in \mathbb{Z}^{\mathcal{C}}$, respectively. Observe that by Strong Linear Programming Duality, $\tau(\mathcal{C}, w) \geq \tau^{\star}(\mathcal{C}, w)=\nu^{\star}(\mathcal{C}, w) \geq \nu(\mathcal{C}, w)$.

Notice the correspondence between the $0-1$ feasible solutions of $(P)$ and the covers of $\mathcal{C}$, as well as the correspondence between the integer feasible solutions of $(D)$ for $w=\mathbf{1}$ and the packings of $\mathcal{C}$. In particular, 
$\tau(\mathcal{C}, \mathbf{1})=\tau(\mathcal{C})$ and $\nu(\mathcal{C}, \mathbf{1})=\nu(\mathcal{C})$. We will refer to the feasible solutions of $(\mathrm{P})$ as fractional covers, and to the feasible solutions of (D) for $w=\mathbf{1}$ as fractional packings.

$\mathcal{C}$ has the max-flow min-cut property if $\tau(\mathcal{C}, w)=\nu(\mathcal{C}, w)$ for all $w \in \mathbb{Z}_{+}^{V}[10] . \mathcal{C}$ is ideal if $\tau(\mathcal{C}, w)=$ $\nu^{\star}(\mathcal{C}, w)$ for all $w \in \mathbb{Z}_{+}^{V}$ [11]. Clearly clutters with the max-flow min-cut property are ideal. The max-flow min-cut property is not closed under taking blockers, but

Theorem 2 ([18]). A clutter is ideal if, and only if, its blocker is ideal.

If $\mathcal{C}$ is an identically self-blocking clutter different from $\{\{a\}\}$, then $\tau(\mathcal{C}) \geq 2>1=\nu(\mathcal{C})$ by Theorem 1 , so $\mathcal{C}$ does not have the max-flow min-cut property. In this paper, we prove the following stronger statement:

Theorem 3. An identically self-blocking clutter different from $\{\{a\}\}$ is nonideal.

For an integer $n \geq 3$, denote by $\Delta_{n}$ the clutter over ground set $\{1, \ldots, n\}$ whose members are $\{1,2\}$, $\{1,3\}, \ldots,\{1, n\},\{2,3, \ldots, n\}$. Notice that the elements and members of $\Delta_{n}$ correspond to the points and lines of a degenerate projective plane. Denote by $\mathbb{L}_{7}$ the clutter over ground set $\{1, \ldots, 7\}$ whose members are $\{1,2,3\},\{1,4,5\},\{1,6,7\},\{2,4,7\},\{2,5,6\},\{3,4,6\},\{3,5,7\}$. Notice that the elements and members of $\mathbb{L}_{7}$ correspond to the points and lines of the Fano plane. It can be readily checked that $\left\{\Delta_{n}: n \geq 3\right\} \cup\left\{\mathbb{L}_{7}\right\}$ are identically self-blocking clutters. There are many other examples of identically self-blocking clutters, and in fact there is one for every pair of blocking clutters ([4], Remark 3.4 and Corollary 3.6). Another example, for instance, is the clutter over ground set $\{1, \ldots, 6\}$ whose members are $\{6,1,2\},\{6,2,3\},\{6,3,4\},\{6,4,5\}$, $\{6,5,1\},\{1,2,4\},\{2,3,5\},\{3,4,1\},\{4,5,2\},\{5,1,3\}$.

Conjecture 4. An identically self-blocking clutter different from $\{\{a\}\}$ has one of $\left\{\Delta_{n}: n \geq 3\right\}, \mathbb{L}_{7},\{\{1,2\}$, $\{2,3\},\{3,4\},\{4,5\},\{5,1\}\}$ as minor.

(Notice that the last clutter above is not identically self-blocking.) For disjoint $X, Y \subseteq V$, the minor of $\mathcal{C}$ obtained after deleting $X$ and contracting $Y$ is the clutter over ground set $V-(X \cup Y)$ whose members are

$$
\mathcal{C} \backslash X / Y:=\text { the inclusionwise minimal sets of }\{C-Y: C \in \mathcal{C}, C \cap X=\emptyset\} \text {. }
$$

It is well-known that $b(\mathcal{C} \backslash X / Y)=b(\mathcal{C}) / X \backslash Y$ [21], and that if a clutter is ideal, then so is every minor of it [22]. It can be readily checked that the clutters in Conjecture 4 are nonideal. Thus Conjecture $4-$ if true would be a strengthening of Theorem 3 .

The rest of the paper is organized as follows: We will present two proofs of Theorem 3, one will be short and indirect $(\S 2)$ while the other will be a longer and direct proof that essentially unravels the first proof $(\S 5)$. In $\S 3$, by using our techniques, we will provide a new lower bound for the packing number of an arbitrary clutter, and in $\S 4$, we will see a surprising emergence of cuboids, a special class of clutters. In $\S 6$ we will address the relevance of studying identically self-blocking clutters, a relatively narrow problem, and why it may be of interest to the community. 


\section{Gauge duality}

Here we present a short and indirect proof of Theorem 3. Take an integer $n \geq 1$ and let $M$ be a matrix with $n$ columns and nonnegative entries and without a row of all zeros. Consider the polyhedron $P:=$ $\left\{x \in \mathbb{R}_{+}^{n}: M x \geq \mathbf{1}\right\}$. The blocker of $P$ is the polyhedron $Q:=\left\{z \in \mathbb{R}_{+}^{n}: z^{\top} x \geq 1 \forall x \in P\right\}$. Fulkerson showed that there exists a matrix $N$ with $n$ columns and nonnegative entries and without a row of all zeros such that $Q=\left\{z \in \mathbb{R}_{+}^{n}: N z \geq \mathbf{1}\right\}$, and that the blocker of $Q$ is $P[15,14]$. In 1987 Chaiken proved the following fascinating result:

Theorem 5 ([8]). Take an integer $n \geq 1$, let $P, Q$ be a blocking pair of polyhedra in $\mathbb{R}^{n}$, and let $R$ be a positive definite $n$ by n matrix. Then $\min \left\{x^{\top} R x: x \in P\right\}$ and $\min \left\{z^{\top} R^{-1} z: z \in Q\right\}$ have reciprocal optimal values.

Theorem 5 exhibits an instance of gauge duality, a general framework introduced by Freund later the same year [13]. Theorem 5 in the special case of diagonal $R$ 's was also proved by Lovász in 2001 [19]. Both Freund and Lovász seem to have been unaware of Chaiken's result.

Let $\mathcal{C}$ be a clutter over ground set $V$. Define the incidence matrix of $\mathcal{C}$ as the matrix $M$ whose columns are indexed by the elements and whose rows are the incidence vectors of the members, and define $Q(\mathcal{C}):=$ $\left\{x \in \mathbb{R}_{+}^{V}: M x \geq \mathbf{1}\right\}$. Fulkerson showed that if $\mathcal{C}, \mathcal{B}$ are blocking ideal clutters then $Q(\mathcal{C}), Q(\mathcal{B})$ give an instance of blocking polyhedra [15, 14]. Therefore Theorem 5 has the following consequence:

Theorem 6. Let $\mathcal{C}, \mathcal{B}$ be blocking ideal clutters. Then $\min \left\{x^{\top} x: x \in Q(\mathcal{C})\right\}$ and $\min \left\{z^{\top} z: z \in Q(\mathcal{B})\right\}$ have reciprocal optimal values.

We will need the following lemma whose proof makes use of concepts such as the Lagrangian and the Karush-Kuhn-Tucker conditions (see [7], Chapter 5):

Lemma 7 ([8]). Let $\mathcal{C}$ be a clutter over ground set $V$, and let $M$ be its incidence matrix. Then $\min \left\{x^{\top} x\right.$ : $M x \geq \mathbf{1}, x \geq \mathbf{0}\}$ has a unique optimal solution $x^{\star} \in \mathbb{R}_{+}^{V}$. Moreover, there exists $y \in \mathbb{R}_{+}^{\mathcal{C}}$ such that $M^{\top} y=x^{\star}$, $\mathbf{1}^{\top} y=x^{\star} x^{\star}$ and $y^{\top}\left(M x^{\star}-\mathbf{1}\right)=\mathbf{0}$.

Proof. Notice that $\min \left\{x^{\top} x: M x \geq \mathbf{1}, x \geq \mathbf{0}\right\}$ satisfies Slater's condition, that there is a feasible solution satisfying all the inequalities strictly. As $x^{\top} x$ is a strictly convex function, our quadratic program has a unique optimal solution $x^{\star} \in \mathbb{R}_{+}^{V}$. Denote by $L(x ; \mu, \sigma):=x^{\top} x-\mu^{\top}(M x-\mathbf{1})-\sigma^{\top} x$ the Lagrangian of the program. Since Slater's condition is satisfied, there exist $\mu^{\star} \in \mathbb{R}_{+}^{\mathcal{C}}$ and $\sigma^{\star} \in \mathbb{R}_{+}^{V}$ satisfying the Karush-Kuhn-Tucker conditions:

$$
\begin{aligned}
& \mathbf{0}=\nabla_{x} L\left(x^{\star} ; \mu^{\star}, \sigma^{\star}\right)=2 x^{\star}-M^{\top} \mu^{\star}-\sigma^{\star} \\
& 0=\mu^{\star \top}\left(M x^{\star}-\mathbf{1}\right) \\
& 0=\sigma^{\star \top} x^{\star} .
\end{aligned}
$$

Let $y:=\frac{1}{2} \mu^{\star}$. Since $\sigma^{\star}$ and $M$ have nonnegative entries, and the third equation holds, the first equation implies that $M^{\top} y=x^{\star}$. Multiplying the first equation by $x^{\star}$ from the left, and taking the next two equations into account, we get that $\mathbf{1}^{\top} y=x^{\star} x^{\star}$. As $y^{\top}\left(M x^{\star}-\mathbf{1}\right)=\mathbf{0}$ clearly holds, $y$ is the desired assignment. 
We are now ready for the first, short and indirect proof of Theorem 3, stating that an identically self-blocking clutter different from $\{\{a\}\}$ is nonideal:

Proof of Theorem 3. Let $\mathcal{C}$ be an identically self-blocking clutter over ground set $V$ that is different from $\{\{a\}\}$, and let $M$ be its incidence matrix. Suppose for a contradiction that $\mathcal{C}$ is ideal. Then Theorem 6 applies and tells us that $\min \left\{x^{\top} x: M x \geq \mathbf{1}, x \geq \mathbf{0}\right\}=1$. Let $x^{\star}, y$ be as in Lemma 7; so $x^{\star}=M^{\top} y$ and $1=x^{\star} x^{\star}=\mathbf{1}^{\top} y$. As $\mathcal{C}$ is an identically self-blocking clutter different from $\{\{a\}\}$, every member has cardinality at least two, and by Theorem 1 every two members intersect, implying in turn that $M M^{\top} \geq J+I .{ }^{1}$ As a result,

$$
1=x^{\star} x^{\star}=y^{\top} M M^{\top} y \geq y^{\top}(J+I) y=y^{\top} \mathbf{1 1}^{\top} y+y^{\top} y=1+y^{\top} y,
$$

implying in turn that $y=\mathbf{0}$, a contradiction.

\section{Lower bounding the packing number}

Here we present a lower bound on the packing number of an arbitrary clutter. We need the following lemma from 1965 proved by Motzkin and Straus:

Lemma 8 ([20]). Let $G=(V, E)$ be a simple graph, and let $L$ be its $V$ by $V$ adjacency matrix:

$$
L_{u v}=\left\{\begin{array}{ll}
1 & \text { if }\{u, v\} \in E \\
0 & \text { otherwise }
\end{array} \quad \forall u, v \in V .\right.
$$

Then

$$
\max \left\{y^{\top} L y: \mathbf{1}^{\top} y=1, y \geq \mathbf{0}\right\}=1-\frac{1}{\omega(G)}
$$

where $\omega(G)$ is the maximum cardinality of a clique of $G$.

Let $\mathcal{C}$ be a clutter over ground set $V$. Finding $\nu(\mathcal{C})$ can be cast as finding the maximum cardinality of a clique of a graph. This observation, combined with Lemma 8 , has the following consequence:

Lemma 9. Let $\mathcal{C}$ be a clutter over ground set $V$, and let $M$ be its incidence matrix. Then

$$
\min \left\{y^{\top} M M^{\top} y-\sum_{C \in \mathcal{C}}(|C|-1) y_{C}^{2}: \mathbf{1}^{\top} y=1, y \geq \mathbf{0}\right\}=\frac{1}{\nu(\mathcal{C})} .
$$

Proof. $(\leq)$ Let $y \in \mathbb{R}_{+}^{\mathcal{C}}$ be the incidence vector of a maximum packing of $\mathcal{C}$. Then $\frac{1}{\nu(\mathcal{C})} \cdot y$ is a feasible solution whose objective value is $\frac{1}{\nu(\mathcal{C})}$, implying in turn that $\leq$ holds. $(\geq)$ Let $G$ be the graph whose vertices correspond to the members of $\mathcal{C}$, where two vertices are adjacent if the corresponding members are disjoint. Let $L$ be the adjacency matrix of $G$. Then $M M^{\top} \geq \operatorname{Diag}(|C|-1: C \in \mathcal{C})+J-L$. Notice that there is a bijection between the packings in $\mathcal{C}$ and the cliques in $G$, and in particular that $\nu(\mathcal{C})=\omega(G)$. Thus by Lemma 8 , for any $y \in \mathbb{R}_{+}^{\mathcal{C}}$ such that $\mathbf{1}^{\top} y=1$,

$$
1-\frac{1}{\nu(\mathcal{C})} \geq y^{\top} L y \geq \sum_{C \in \mathcal{C}}(|C|-1) y_{C}^{2}+y^{\top} J y-y^{\top} M M^{\top} y=\sum_{C \in \mathcal{C}}(|C|-1) y_{C}^{2}+1-y^{\top} M M^{\top} y,
$$

\footnotetext{
${ }^{1}$ Throughout the paper, $J$ is a square all ones matrix of appropriate dimension, and $I$ is the identity matrix of appropriate dimension.
} 
implying in turn that $\geq$ holds.

As a consequence, after employing Carathéodory's theorem (see [9], §3.14) and the Cauchy-Schwarz inequality (see [23]), we get the following lower bound on the packing number of a clutter:

Theorem 10 ([5]). Let $\mathcal{C}$ be a clutter over ground set $V$, and let $M$ be its incidence matrix. Then

$$
\nu(\mathcal{C}) \geq\left(\frac{y^{\top} M M^{\top} y}{y^{\top} J y}-\frac{\min \{|C|-1: C \in \mathcal{C}\}}{\min \{|V|,|\mathcal{C}|\}}\right)^{-1} \forall y \in \mathbb{R}_{+}^{\mathcal{C}}, y \neq \mathbf{0} .
$$

Proof. Pick a nonzero $y \in \mathbb{R}_{+}^{\mathcal{C}}$. By Carathéodory's theorem there is a $y^{\prime} \in \mathbb{R}_{+}^{\mathcal{C}}$ such that $M^{\top} y^{\prime} \leq M^{\top} y$, $\mathbf{1}^{\top} y^{\prime}=\mathbf{1}^{\top} y$ and $\left|\operatorname{supp}\left(y^{\prime}\right)\right| \leq|V|$. Lemma 9 applied to $\frac{1}{\mathbf{1}^{\top} y^{\prime}} \cdot y^{\prime}$ implies that

$$
\nu(\mathcal{C}) \geq\left(\frac{y^{\prime \top} M M^{\top} y^{\prime}-\sum_{C \in \mathcal{C}}(|C|-1) y_{C}^{\prime 2}}{y^{\prime} J y^{\prime}}\right)^{-1} \geq\left(\frac{y^{\top} M M^{\top} y}{y^{\top} J y}-\frac{\sum_{C \in \mathcal{C}}(|C|-1) y_{C}^{\prime 2}}{y^{\prime \top} J y^{\prime}}\right)^{-1} .
$$

By the Cauchy-Schwarz inequality applied to the nonzero entries of $y^{\prime}$,

$$
\frac{\sum_{C \in \mathcal{C}}(|C|-1) y_{C}^{\prime 2}}{y^{\prime \top} J y^{\prime}} \geq \frac{\left(\sum_{C \in \mathcal{C}} \sqrt{|C|-1} \cdot y_{C}^{\prime}\right)^{2}}{\left|\operatorname{supp}\left(y^{\prime}\right)\right| \cdot y^{\prime \top} J y^{\prime}} \geq \frac{\min \{|C|-1: C \in \mathcal{C}\}}{\min \{|V|,|\mathcal{C}|\}} .
$$

Combining the last two inequalities proves the theorem.

This theorem was proved implicitly by Aharoni, Erdôs and Linial in 1988. Given that $M$ is the incidence matrix of $\mathcal{C}$, the authors explicitly proved Theorem 10 for $y$ a maximum fractional packing of $\mathcal{C}$ :

$$
\nu(\mathcal{C}) \geq\left(\frac{\mathbf{1}^{\top} \mathbf{1}}{\nu^{\star 2}(\mathcal{C})}-\frac{\min \{|C|-1: C \in \mathcal{C}\}}{\min \{|V|,|\mathcal{C}|\}}\right)^{-1} \geq \frac{\nu^{\star 2}(\mathcal{C})}{|V|} .
$$

But one can do better:

Theorem 11. Let $\mathcal{C}$ be a clutter over ground set $V$, and let $\alpha:=\min \left\{x^{\top} x: x \in Q(\mathcal{C})\right\}$. Then

$$
\nu(\mathcal{C}) \geq\left(\frac{1}{\alpha}-\frac{\min \{|C|-1: C \in \mathcal{C}\}}{\min \{|V|,|\mathcal{C}|\}}\right)^{-1} .
$$

Proof. Let $M$ be the incidence matrix of $\mathcal{C}$, and let $x \in \mathbb{R}_{+}^{V}$ be the point in $Q(\mathcal{C})$ such that $x^{\top} x=\alpha$. By Lemma 7, there exists $y \in \mathbb{R}_{+}^{\mathcal{C}}$ such that $x=M^{\top} y$ and $\mathbf{1}^{\top} y=\alpha$. By Theorem 10,

$$
\nu(\mathcal{C}) \geq\left(\frac{x^{\top} x}{y^{\top} \mathbf{1 1}^{\top} y}-\frac{\min \{|C|-1: C \in \mathcal{C}\}}{\min \{|V|,|\mathcal{C}|\}}\right)^{-1}=\left(\frac{\alpha}{\alpha^{2}}-\frac{\min \{|C|-1: C \in \mathcal{C}\}}{\min \{|V|,|\mathcal{C}|\}}\right)^{-1},
$$

as required.

Let

$$
\beta:=\min \left\{\frac{y^{\top} M M^{\top} y}{y^{\top} J y}: y \geq \mathbf{0}, y \neq \mathbf{0}\right\} \quad \text { and } \quad \alpha:=\min \left\{x^{\top} x: M x \geq \mathbf{1}, x \geq \mathbf{0}\right\} .
$$

By Strong Conic Programming Duality (see [7], Chapter 5),

$$
\frac{1}{\sqrt{\beta}}=\max \left\{\mathbf{1}^{\top} y:\left\|M^{\top} y\right\| \leq 1, y \geq \mathbf{0}\right\}=\min \{\|x\|: M x \geq \mathbf{1}, x \geq \mathbf{0}\}=\sqrt{\alpha},
$$


so $\beta=\frac{1}{\alpha}$. As a result, the inequality given by Theorem 11 is the best lower bound derived from Theorem 10 .

As an immediate consequence of Theorem 11, we get another new lower bound on the packing number of a clutter:

Theorem 12. Let $\mathcal{C}$ be a clutter. Then $\nu(\mathcal{C}) \geq \min \left\{x^{\top} x: x \in Q(\mathcal{C})\right\}$.

See ([1], Chapter 3, Theorem 3.2) for an alternative proof of this theorem.

\section{Cuboids}

Take an even integer $n \geq 2$. A cuboid is a clutter over ground set $\{1, \ldots, n\}$ where every member $C$ satisfies $|C \cap\{1,2\}|=|C \cap\{3,4\}|=\cdots=|C \cap\{n-1, n\}|=1$. Introduced in [2], cuboids form a very special class of clutters, to the point that some of the main conjectures in the field can be phrased equivalently in terms of cuboids [3]. Cuboids play a special role here also:

Theorem 13. Let $\mathcal{C}$ be an ideal clutter over n elements whose members do not have a common element, and let $\alpha:=\min \left\{x^{\top} x: x \in Q(\mathcal{C})\right\}$. Then $\alpha \geq \frac{4}{n}$. Moreover, the following statements are equivalent:

(i) $\alpha=\frac{4}{n}$,

(ii) $n$ is even, after a possible relabeling of the ground set the sets $\{1,2\},\{3,4\}, \ldots,\{n-1, n\}$ are minimal covers, and the members of minimum cardinality form an ideal cuboid over ground set $\{1, \ldots, n\}$ whose members do not have a common element.

To prove this theorem we need a few preliminary results. Given a simple graph $G=(V, E)$, a fractional perfect matching is a $y \in \mathbb{R}_{+}^{E}$ such that $\mathbf{1}^{\top} y=\frac{|V|}{2}$ and for each vertex $u \in V, \sum\left(y_{e}: u \in e\right)=1$. We need the following classic result:

Lemma 14 (folklore). If a bipartite graph has a fractional perfect matching, then it has a perfect matching.

Lemma 15. Take an integer $n \geq 2$, and let $\mathcal{C}$ be a clutter over ground set $\{1, \ldots, n\}$. Then the following statements are equivalent:

(i) $\mathcal{C}$ has a fractional packing of value 2 and $b(\mathcal{C})$ has a fractional packing of value $\frac{n}{2}$,

(ii) $n$ is even, and in $\mathcal{C}$, after a possible relabeling of the ground set the sets $\{1,2\},\{3,4\}, \ldots,\{n-1, n\}$ are minimal covers, and the members of minimum cardinality form a cuboid over ground set $\{1, \ldots, n\}$ with a fractional packing of value 2.

Proof. (ii) $\Rightarrow$ (i) is immediate. (i) $\Rightarrow$ (ii): Let $M, N$ be the incidence matrices of $\mathcal{C}, b(\mathcal{C})$, respectively. Let $y \in \mathbb{R}_{+}^{\mathcal{C}}$ be a fractional packing of $\mathcal{C}$ of value 2 ; so $M^{\top} y \leq \mathbf{1}$ and $\mathbf{1}^{\top} y=2$. Let $t \in \mathbb{R}_{+}^{b(\mathcal{C})}$ be a fractional packing of $b(\mathcal{C})$ of value $\frac{n}{2}$; so $N^{\top} t \leq \mathbf{1}$ and $\mathbf{1}^{\top} t=\frac{n}{2}$. Then $n=\mathbf{1}^{\top} \mathbf{1} \geq t^{\top} N M^{\top} y \geq t^{\top} J y=t^{\top} \mathbf{1 1}^{\top} y=\frac{n}{2} \cdot 2=n$. Thus equality holds throughout, implying in turn that 
(1) $M^{\top} y=\mathbf{1}$ and $\mathbf{1}^{\top} y=2$,

(2) $N^{\top} t=\mathbf{1}$ and $\mathbf{1}^{\top} t=\frac{n}{2}$,

(3) if $y_{C}>0$ and $t_{B}>0$ for some $C \in \mathcal{C}$ and $B \in b(\mathcal{C})$, then $|C \cap B|=1$.

Notice that $\tau(\mathcal{C}) \geq 2$ and $\tau(b(\mathcal{C})) \geq \frac{n}{2}$, so every member of $\mathcal{C}$ has cardinality at least $\frac{n}{2}$ while every member of $b(\mathcal{C})$ has cardinality at least 2 . Together with (1) and (2), these observations imply that $n$ is even, and

(4) if $y_{C}>0$ for some $C \in \mathcal{C}$, then $|C|=\frac{n}{2}$,

(5) if $t_{B}>0$ for some $B \in b(\mathcal{C})$, then $|B|=2$.

Let $G$ be the graph over vertices $\{1, \ldots, n\}$ whose edges correspond to $\left\{B \in b(\mathcal{C}): t_{B}>0\right\}$. Pick $C \in \mathcal{C}$ such that $y_{C}>0$. Then by (3) the vertex subset $C$ intersects every edge of $G$ exactly once, implying in turn that $G$ is a bipartite graph. By (2) $G$ has a fractional perfect matching, and as the graph is bipartite, there must be a perfect matching by Lemma 14 , labeled as $\{1,2\},\{3,4\}, \ldots,\{n-1, n\}$ after a possible relabeling of the ground set. As a consequence, the members of $\mathcal{C}$ of minimum cardinality form a cuboid over ground set $\{1, \ldots, n\}$ which by (1) and (4) has a fractional packing of value 2 . Thus (ii) holds.

Remark 16. Let $\mathcal{C}$ be a clutter over $n$ elements, and let $\alpha, x^{\star}$ be the optimal value and solution of $\min \left\{x^{\top} x\right.$ : $x \in Q(\mathcal{C})\}$, respectively. Then the following statements hold:

(i) $\alpha \geq \frac{\nu^{\star 2}(\mathcal{C})}{n}$. Moreover, equality holds if and only if $x^{\star}=\frac{\nu^{\star}(\mathcal{C})}{n} \cdot \mathbf{1}$.

(ii) Assume that every member has cardinality at least two. Then $\alpha \leq \frac{n}{4}$. Moreover, equality holds if and only if $x^{\star}=\frac{1}{2} \cdot \mathbf{1}$.

Proof. (i) The Cauchy-Schwarz inequality implies that $\alpha=x^{\star \top} x^{\star} \geq \frac{\left(\mathbf{1}^{\top} x^{\star}\right)^{2}}{n} \geq \frac{\tau^{\star 2}(\mathcal{C})}{n}=\frac{\nu^{\star 2}(\mathcal{C})}{n}$. Moreover, equality holds throughout if and only if the entries of $x^{\star}$ are equal and $\mathbf{1}^{\top} x^{\star}=\nu^{\star}(\mathcal{C})$, i.e. $x^{\star}=\frac{\nu^{\star}(\mathcal{C})}{n} \cdot \mathbf{1}$. (ii) is immediate.

We also need the following result proved implicitly in [2] (its proof can be found in the proof of Theorem 1.6, Claim 3 on page 543):

Lemma 17 ([2]). Take an even integer $n \geq 2$, and let $\mathcal{C}$ be an ideal clutter over ground set $\{1, \ldots, n\}$ where $\{1,2\},\{3,4\}, \ldots,\{n-1, n\}$ are minimal covers. Then $\left\{C \in \mathcal{C}:|C|=\frac{n}{2}\right\}$ is an ideal cuboid.

We are now ready to prove Theorem 13:

Proof of Theorem 13. By Remark 16 (i), $\alpha \geq \frac{\nu^{\star 2}(\mathcal{C})}{n}=\frac{\tau^{2}(\mathcal{C})}{n} \geq \frac{4}{n}$, where the equality follows from the fact that $\mathcal{C}$ is ideal, and the last inequality holds because the members have no common element. (i) $\Rightarrow$ (ii): Assume that $\alpha=\frac{4}{n}$. Let $x^{\star}$ be the optimal solution of $\min \left\{x^{\top} x: x \in Q(\mathcal{C})\right\}$. Then by Remark 16 (i), $\tau(\mathcal{C})=2$ and $x^{\star}=\left(\frac{2}{n}, \frac{2}{n}, \ldots, \frac{2}{n}\right)$. Let $M$ be the incidence matrix of $\mathcal{C}$. By Lemma 7 , there is a $y \in \mathbb{R}_{+}^{\mathcal{C}}$ such that $M^{\top} y=x^{\star}$ and $\mathbf{1}^{\top} y=\frac{4}{n}$, that is, 
$\frac{n}{2} \cdot y$ is a fractional packing of $\mathcal{C}$ of value 2.

Let $\beta, z^{\star}$ be the optimal value and solution of $\min \left\{z^{\top} z: z \in Q(b(\mathcal{C}))\right\}$. As $\mathcal{C}$ is an ideal clutter, it follows from Theorem 6 that $\beta=\frac{1}{\alpha}=\frac{n}{4}$. Thus by Remark 16 (ii), $z^{\star}=\left(\frac{1}{2}, \frac{1}{2}, \ldots, \frac{1}{2}\right)$. Let $N$ be the incidence matrix of $b(\mathcal{C})$. By Lemma 7, there is a $t \in \mathbb{R}_{+}^{b(\mathcal{C})}$ such that $N^{\top} t=z^{\star}$ and $\mathbf{1}^{\top} z^{\star}=\frac{n}{4}$, that is,

$2 t$ is a fractional packing of $b(\mathcal{C})$ of value $\frac{n}{2}$.

It therefore follows from Lemma 15 that $n$ is even, after a possible relabeling of the ground set the sets $\{1,2\},\{3,4\}, \ldots,\{n-1, n\}$ are minimal covers of $\mathcal{C}$, and the members of $\mathcal{C}$ of minimum cardinality form a cuboid $\mathcal{C}_{0}$ over ground set $\{1, \ldots, n\}$ with a fractional packing of value 2 . In particular, the members of $\mathcal{C}_{0}$ do not have a common element. Moreover, since $\mathcal{C}$ is an ideal clutter, it follows from Lemma 17 that $\mathcal{C}_{0}$ is an ideal cuboid, thereby proving (ii). (ii) $\Rightarrow$ (i): Observe that every member has cardinality at least $\frac{n}{2}$, so $\left(\frac{2}{n}, \frac{2}{n}, \ldots, \frac{2}{n}\right) \in Q(\mathcal{C})$, implying in turn that $\alpha \leq \frac{4}{n}$. Since $\alpha \geq \frac{4}{n}$ also, (i) must hold.

We showed that among ideal clutters $\mathcal{C}$ whose members do not have a common element, it is essentially cuboids that achieve the smallest possible value for $\min \left\{x^{\top} x: x \in Q(\mathcal{C})\right\}$. Our proof relied on Lemma 15, which in itself has another consequence. Viewing clutters as simple games, Hof et al. [16] showed that given a clutter $\mathcal{C}$ over $n$ elements, its critical threshold value is always at most $\frac{n}{4}$, and this maximum is achieved if, and only if, $\mathcal{C}$ has a fractional packing of value $\frac{n}{2}$ and $b(\mathcal{C})$ has a fractional packing of value 2 . Thus by Lemma 15 , it is essentially blockers of cuboids that achieve the largest possible critical threshold value.

\section{Bypassing gauge duality}

Here we present a longer and direct proof of the main result of the paper, Theorem 3. This proof will bypass the use of Theorem 6. We will need the following two lemmas:

Lemma 18. Let $\mathcal{C}, \mathcal{B}$ be clutters over ground set $V$ such that $|C \cap B|=1$ for all $C \in \mathcal{C}, B \in \mathcal{B}$, for which there exist nonzero $y \in \mathbb{R}_{+}^{\mathcal{C}}$ and $t \in \mathbb{R}_{+}^{\mathcal{B}}$ such that $\sum_{C \in \mathcal{C}} y_{C} \chi_{C}=\sum_{B \in \mathcal{B}} t_{B} \chi_{B}$. Then

$$
\nu(\mathcal{C}) \geq\left(\frac{\mathbf{1}^{\top} t}{\mathbf{1}^{\top} y}-\frac{\min \{|C|-1: C \in \mathcal{C}\}}{\min \{|V|,|\mathcal{C}|\}}\right)^{-1} \quad \text { and } \quad \nu(\mathcal{B}) \geq\left(\frac{\mathbf{1}^{\top} y}{\mathbf{1}^{\top} t}-\frac{\min \{|B|-1: B \in \mathcal{B}\}}{\min \{|V|,|\mathcal{B}|\}}\right)^{-1} .
$$

Proof. Due to the symmetry between $\mathcal{C}$ and $\mathcal{B}$, it suffices to prove the first inequality. After possibly scaling $t$, we may assume that $\mathbf{1}^{\top} t=1$. Our hypotheses imply that for each $C^{\prime} \in \mathcal{C}$,

$$
\sum_{C \in \mathcal{C}} y_{C}\left|C^{\prime} \cap C\right|=\sum_{C \in \mathcal{C}} y_{C} \chi_{C^{\prime}}^{\top} \chi_{C}=\sum_{B \in \mathcal{B}} t_{B} \chi_{C^{\prime}}^{\top} \chi_{B}=\sum_{B \in \mathcal{B}} t_{B}\left|C^{\prime} \cap B\right|=1 .
$$

Thus, given that $M$ is the incidence matrix of $\mathcal{C}$, the equalities above state that $M M^{\top} y=1$. And Theorem 10 applied to $y$ implies that

$$
\nu(\mathcal{C}) \geq\left(\frac{y^{\top} \mathbf{1}}{y^{\top} J y}-\frac{\min \{|C|-1: C \in \mathcal{C}\}}{\min \{|V|,|\mathcal{C}|\}}\right)^{-1},
$$

therefore implying the first inequality. 
Given a clutter, a fractional cover is minimal if it is not greater than or equal to another fractional cover. Given an ideal clutter, it is well-known that every minimal fractional cover can be written as a convex combination of the incidence vectors of minimal covers (see for instance [11]). We will use this fact below:

Lemma 19. Let $\mathcal{C}, \mathcal{B}$ be blocking ideal clutters. Then there exist nonempty $\mathcal{C}^{\prime} \subseteq \mathcal{C}$ and $\mathcal{B}^{\prime} \subseteq \mathcal{B}$ such that $|C \cap B|=1$ for all $C \in \mathcal{C}^{\prime}, B \in \mathcal{B}^{\prime}$, for which there exist nonzero $y \in \mathbb{R}_{+}^{\mathcal{C}^{\prime}}$ and $t \in \mathbb{R}_{+}^{\mathcal{B}^{\prime}}$ such that $\sum_{C \in \mathcal{C}^{\prime}} y_{C} \chi_{C}=$ $\sum_{B \in \mathcal{B}^{\prime}} t_{B} \chi_{B}$

Proof. Let $M, N$ be the incidence matrices of $\mathcal{C}, \mathcal{B}$, respectively. Let $x^{\star}$ be the optimal solution of $\min \left\{x^{\top} x\right.$ : $x \in Q(\mathcal{C})\}$. Then $x^{\star}$ is a minimal fractional cover of $\mathcal{C}$. As $\mathcal{C}$ is an ideal clutter, $x^{\star}=N^{\top} t$ for some $t \in \mathbb{R}_{+}^{\mathcal{B}}$ such that $\mathbf{1}^{\top} t=1$. Moreover, by Lemma 7, there exists $y \in \mathbb{R}_{+}^{\mathcal{C}}$ such that $M^{\top} y=x^{\star}$ and $y^{\top}\left(M x^{\star}-\mathbf{1}\right)=\mathbf{0}$. Thus, $\mathbf{1}^{\top} y=x^{\star \top} M^{\top} y=t^{\top} N M^{\top} y \geq t^{\top} J y=t^{\top} \mathbf{1 1}^{\top} y=\mathbf{1}^{\top} y$, implying that $t^{\top} N M^{\top} y=t^{\top} J y$. Therefore, if $\mathcal{C}^{\prime}:=\left\{C \in \mathcal{C}: y_{C}>0\right\}$ and $\mathcal{B}^{\prime}:=\left\{B \in \mathcal{B}: t_{B}>0\right\}$, we have that $|C \cap B|=1$ for all $C \in \mathcal{C}^{\prime}, B \in \mathcal{B}^{\prime}$. Moreover, the equation $M^{\top} y=x^{\star}=N^{\top} t$ implies that $\sum_{C \in \mathcal{C}^{\prime}} y_{C} \chi_{C}=\sum_{B \in \mathcal{B}^{\prime}} t_{B} \chi_{B}$. As $\mathcal{C}^{\prime}$ and $\mathcal{B}^{\prime}$ are clearly nonempty, they are the desired clutters.

We are now ready to prove the following statement:

Theorem 20. Let $\mathcal{C}, \mathcal{B}$ be blocking ideal clutters. If $\tau(\mathcal{C}) \geq 2$ and $\tau(\mathcal{B}) \geq 2$, then $\nu(\mathcal{C}) \geq 2$ or $\nu(\mathcal{B}) \geq 2$.

Proof. Assume that $\tau(\mathcal{C}) \geq 2$ and $\tau(\mathcal{B}) \geq 2$. By Lemma 19, there exist nonempty $\mathcal{C}^{\prime} \subseteq \mathcal{C}$ and $\mathcal{B}^{\prime} \subseteq \mathcal{B}$ such that $|C \cap B|=1$ for all $C \in \mathcal{C}^{\prime}, B \in \mathcal{B}^{\prime}$, for which there exist nonzero $y \in \mathbb{R}_{+}^{\mathcal{C}^{\prime}}$ and $t \in \mathbb{R}_{+}^{\mathcal{B}^{\prime}}$ such that $\sum_{C \in \mathcal{C}^{\prime}} y_{C} \chi_{C}=\sum_{B \in \mathcal{B}^{\prime}} t_{B} \chi_{B}$. As the members of $\mathcal{C}^{\prime}$ and $\mathcal{B}^{\prime}$ have cardinality at least two, we get from Lemma 18 that $\nu\left(\mathcal{C}^{\prime}\right)>\frac{\mathbf{1}^{\top} y}{\mathbf{1}^{\top} t}$ and $\nu\left(\mathcal{B}^{\prime}\right)>\frac{\mathbf{1}^{\top} t}{\mathbf{1}^{\top} y}$. As $\nu(\mathcal{C}) \geq \nu\left(\mathcal{C}^{\prime}\right)$ and $\nu(\mathcal{B}) \geq \nu\left(\mathcal{B}^{\prime}\right)$, it follows that $\nu(\mathcal{C}) \geq 2$ or $\nu(\mathcal{B}) \geq 2$, as required.

We are now ready to prove Theorem 3, stating that an identically self-blocking clutter different from $\{\{a\}\}$ is nonideal:

Another proof of Theorem 3. Let $\mathcal{C}$ be an identically self-blocking clutter different from $\{\{a\}\}$. Then $\tau(\mathcal{C}) \geq 2$, and $\nu(\mathcal{C})=1$ by Theorem 1 . Theorem 20 now applies and tells us that $\mathcal{C}$ cannot be ideal, as required.

\section{Concluding remarks}

Given a general blocking pair $\mathcal{C}, \mathcal{B}$ of ideal clutters, what can be said about them? This is an important research topic in Integer Programming and Combinatorial Optimization. We showed that if $\tau(\mathcal{C}) \geq 2$ and $\tau(\mathcal{B}) \geq 2$, then $\nu(\mathcal{C}) \geq 2$ or $\nu(\mathcal{B}) \geq 2$ (Theorems 3 and 20). Equivalently, if the members of $\mathcal{C}, \mathcal{B}$ have cardinality at least two, then one of the two clutters has a bicoloring, i.e. the ground set can be bicolored so that every member receives an element of each color. Next to Lehman's width-length characterization [18], this is the only other fact known about the structure of $\mathcal{C}$ and $\mathcal{B}$. As such, we expect the results as well as the tools introduced here to help us address the question in mind. 
Our main result led us to two computable lower bounds - one weaker than the other - on the packing number of an arbitrary clutter (Theorems 11 and 12). We believe these lower bounds will have applications beyond the scope of this paper. We also characterized the clutters on which one of the lower bounds is at its weakest; we showed that these clutters are essentially cuboids (Theorem 13). Combined with evidence from [2, 3], this only stresses further the central role of cuboids when studying ideal clutters.

Finally, we used techniques from Convex Optimization to prove the main result of the paper. A natural question is whether there is an elementary and discrete approach for proving the result? Conjecture 4 provides a potential approach and leads to an interesting research direction.

\section{Acknowledgements}

We would like to thank Fatma Kılınç-Karzan, Kanstantsin Pashkovich and Levent Tunçel for fruitful discussions about different parts of this work. We would also like to thank the referees; their feedback improved the presentation of the paper. This work was supported in part by ONR grant 000141812129 and NSF grant CMMI 1560828.

\section{References}

[1] Abdi, A.: Ideal Clutters. Ph.D. Dissertation, University of Waterloo (2018)

[2] Abdi, A., Cornuéjols, G., Pashkovich, K.: Ideal clutters that do not pack. Math. Oper. Res. 43(2), 533-553 (2018)

[3] Abdi, A., Cornuéjols, G., Guričanová, N., Lee, D.: Cuboids, a class of clutters. Submitted.

[4] Abdi, A. and Pashkovich, K.: Delta minors, delta free clutters, and entanglement. SIAM J. Discrete Math. 32(3), 1750-1774 (2018)

[5] Aharoni, R., Erdős, P., Linial, N.: Optima of dual integer linear programs. Combinatorica 8(1), 13-20 (1988)

[6] Berge, C.: Hypergraphs: Combinatorics of Finite Sets. North Holland, Amsterdam (1989)

[7] Boyd, S. and Vandenberghe, L.: Convex Optimization. Cambridge University Press (2004)

[8] Chaiken, S.: Extremal length and width of blocking polyhedra, Kirchhoff spaces and multiport networks. SIAM J. Alg. Disc. Meth. 8(4), 635-645 (1987)

[9] Conforti, M., Cornuéjols, G., Zambelli, G.: Integer Programming. Springer (2014)

[10] Cornuéjols, G.: Combinatorial Optimization, Packing and Covering. SIAM, Philadelphia (2001) 
[11] Cornuéjols, G. and Novick, B.: Ideal 0,1 matrices. J. Combin. Theory Ser. B 60(1), 145-157 (1994)

[12] Edmonds, J. and Fulkerson, D.R.: Bottleneck extrema. J. Combin. Theory Ser. B 8(3), 299-306 (1970)

[13] Freund, R.M.: Dual gauge programs, with applications to quadratic programming and the minimum-norm problem. Math. Program. 38, 47-67 (1987)

[14] Fulkerson, D.R.: Blocking and anti-blocking pairs of polyhedra. Math. Program. 1, 168-194 (1971)

[15] Fulkerson, D.R.: Blocking polyhedra. In Graph Theory and Its Applications (ed. Harris B.). Academic Press, New York, 93-112 (1970)

[16] Hof, F., Kern, W., Kurz, S., Pashkovich, K., Paulusma, D.: Simple games versus weighted voting games: bounding the critical threshold value. Preprint, arXiv:1810.08841 (2018)

[17] Isbell, J.R.: A class of simple games. Duke Math. J. 25(3), 423-439 (1958)

[18] Lehman, A.: On the width-length inequality. Math. Program. 17(1), 403-417 (1979)

[19] Lovász, L.: Energy of convex sets, shortest paths, and resistance. J. Combin. Theory Ser. A 94(2), 363-382 (2001)

[20] Motzkin, T.S. and Straus, E.G.: Maxima for graphs and a new proof of a theorem of Turán. Canad. J. Math. 17, 533-540 (1965)

[21] Seymour, P.D.: The forbidden minors of binary matrices. J. London Math. Society 2(12), 356-360 (1976)

[22] Seymour, P.D.: The matroids with the max-flow min-cut property. J. Combin. Theory Ser. B 23(2-3), 189$222(1977)$

[23] Steele, J.M.: The Cauchy-Schwarz Master Class: An Introduction to the Art of Mathematical Inequalities. Cambridge University Press (2004) 